\title{
Constancy Mechanisms and the Normativity of Perception
}

\section{Forthcoming in Giving a Damn: Essays in Dialogue with John Haugeland, edited by Zed Adams and Jake Browning. MIT Press.}

\author{
Zed Adams (New School for Social Research) \\ and \\ Chauncey Maher (Dickinson College)
}

\begin{abstract}
To recognize something is to respond to it in a way that distinguishes it from other things; to recognize is to tell apart. But differential response cannot be the whole story, for two deeply related reasons. First, what is recognized is always some determinate item, feature, or characteristic of the confronted situation, whereas a given response can equally well be taken as a response to any of several distinct things. Second, recognition, unlike response, is a normative notion: it is possible to misrecognize something, to get it wrong, whereas a response is just whatever response it is to whatever is there. These are related because: only insofar as something determinate is supposed to be recognized, can there be an issue of recognizing it rightly or wrongly; and it is only as that which determines rightness or wrongness that the object of recognition is determinate.
\end{abstract}

-John Haugeland, “Objective Perception” (HT 272)

\section{The Problem of Perceptual Representation}

Imagine that John is scouring the Arizona desert for a scorpion, in order to encase it in resin for the aiguillette on his bolo tie. As he looks under a small rock, he sees something that looks like a scorpion, swinging his net down on it. But it is not a scorpion. It is, in fact, a plastic scorpion left there by a mischievous graduate student. In frustration, he sits down on a large rock, thinking he might just give up wearing bolo ties altogether. As he does so, he suddenly feels a sharp pain in his lower regions, realizing that he has found what he was looking for, albeit unintentionally.

Consider John's visual experience as he looks under the small rock. It makes sense to assess it as accurate or inaccurate, and it turns out that it is inaccurate. Contrast this with John's pain experience as he sits down on the large rock. There are many things we could say about this experience, but it would not make sense to assess it as accurate or inaccurate.

What marks the difference between these two sorts of experience? Why can some be assessed as accurate or inaccurate, whereas others cannot? More generally, what does it take for a sensory experience to represent things as being a certain way, such that it can be assessed in terms of accuracy and inaccuracy? Call this the problem of perceptual representation.

In Origins of Objectivity (2010), Tyler Burge attempts to solve this problem. He proposes that a sensory experience is representational if and only if it is an exercise of a perceptual constancy mechanism. In his preferred terms, a "sensory state" is representational—it is a "perception" or a "perceptual representation"-if and only if it is an exercise of a perceptual 
constancy mechanism. As he puts it, "perceptual constancies are necessary as well as sufficient for perceptual objectification and perceptual representation” (2010, 413).

In this essay, we draw on John Haugeland's work in order to argue that Burge is wrong to think that exercises of perceptual constancy mechanisms suffice for perceptual representation. Although Haugeland did not live to read or respond to Burge's Origins of Objectivity, we think that his work contains resources that can be developed into a critique of the very foundation of Burge's approach. Specifically, we identify two related problems for Burge. First, if (what Burge calls) mere sensory responses are not representational, then neither are exercises of constancy mechanisms, since the differences between them do not suffice to imply that one is representational and the other is not. Second, taken by themselves, exercises of constancy mechanisms are only derivatively representational, so merely understanding how they work is not sufficient for understanding what is required for something, in itself, to be representational (and thereby provide a full solution to the problem of perceptual representation).

In $\S 2$, we offer a concise summary of Burge's account of perceptual representation. (It is worth noting that Burge's own articulation of this account spans 656 pages.) In $\S 3$ we draw upon Haugeland's work to identify and spell out the problems for Burge's account just mentioned. In $\S 4$ we conclude by spelling out why we think the failure of Burge's account is nevertheless a very good starting point for solving the problem of perceptual representation.

\section{Burge on Perceptual Representation}

In this section, we summarize Burge's account of perceptual representation, canvassing his criticisms of two familiar theories of perceptual representation, before presenting his own positive proposal.

\subsubsection{Information-Theoretic Theories of Perceptual Representation}

Information-Theoretic theories (hereafter, IT theories) are based on the idea that sensory states can correlate with or "indicate" states of the environment. ${ }^{1}$ There are different versions of this sort of theory, which vary mainly according to how correlation or indication is understood. ${ }^{2}$ An influential version of the theory emphasizes causation: $\mathrm{X}$ indicates $\mathrm{Y}$ when $\mathrm{X}$ is caused by $\mathrm{Y}^{3}$ Why think this sort of relationship suffices for perceptual representation? Consider an example from outside the realm of sensation. The fossil of a Megalosaurus's femur unearthed in Cornwell in 1676 was caused to be the way it is by a Megalosaurus's femur in that same place 166 million years ago. A number of this fossil's features causally depend upon the femur's features: most

\footnotetext{
${ }^{1}$ We use "indicates" and its cognates roughly as Dretske does in his (1988), which builds on his (1981). As Dretske there observes, indication is essentially the same as "natural meaning" or "meaning" in Grice (1957). We follow Shannon and Weaver (1949) in referring to these sorts of theories as "information-theoretic" theories.

${ }^{2}$ For discussion of the varieties, see (Godfrey-Smith 1992) and (Neander 2004/2012). For our purposes here, we are overlooking the subtle, but important, distinctions between these varieties.

${ }^{3}$ More precisely, a token of type X's being $\mathrm{F}$ indicates a token of type Y's being $\mathrm{G}$ when tokens of type X being $\mathrm{F}$ are caused by tokens of type Y being G. Although it can matter in other contexts, unless we say otherwise, we will generally abstract away from the distinction between types and tokens.
} 
obviously, the fossil's size and shape causally depend on the femur's size and shape. Thus, the size and shape of the fossil indicate - or "carry information about" - the size and shape of the femur. That is a reason one might think of the fossil as a representation of the femur.

An IT theory of perceptual representation holds that indication suffices for representation. Schematically, a sensory state $\mathrm{S}$ of an organism $\mathrm{O}$ represents a state of the environment $\mathrm{E}$ if $\mathrm{S}$ indicates E.

Following many others, Burge alleges that the problem with IT theories is that they do not allow for the possibility of error; they do not allow for misindication. ${ }^{4}$ A sensory state either indicates an actual state of affairs, or it does not indicate anything at all. It is impossible for it to make a mistaken indication. Consider the fossil. Although it can indicate many things about the Megalosaurus's femur, it cannot mistakenly indicate anything about this femur - or anything else for that matter. To misindicate would be to indicate incorrectly, e.g., to indicate that the femur was larger or shaped differently than it actually was. However, a property of the fossil can indicate only whatever it was that actually caused the fossil to have that property. Consequently, misindication is impossible. For this reason, IT theories cannot explain the possibility of misrepresentation, which is a necessary part of explaining the possibility of representation. ${ }^{5}$

Of course, we can misunderstand what the fossil indicates and thereby come to have false beliefs about the Megalosaurus. For instance, we might mistakenly take part of the rock surrounding the fossil to be part of the fossil, and thereby come to believe that the Megalosaurus's femur was larger than it actually was. So, there is a sense in which fossils can misrepresent: namely, we can take them to misrepresent something. But this misrepresentation is our mistake. In general, the only way in which a fossil can misrepresent is in the derivative sense that something that already has representations can take the fossil to indicate something that it does not. But fossils themselves do not misrepresent anything.

\subsubsection{Teleosemantic Theories of Perceptual Representation}

Teleosemantic theories (hereafter, TS theories) attempt to improve on IT theories by making room for misrepresentation. They appeal to the idea of a biologically evolved function. Roughly, misrepresentation is a form of malfunction. If $\mathrm{X}$ has the function of indicating $\mathrm{Y}$, then, if $\mathrm{X}$ malfunctions, it misindicates $\mathrm{Y}$.

What is it for something to have a biologically evolved function? ${ }^{6}$ According to the most prominent versions of TS theories, the function of a trait is what that trait has been selected for. A trait gets selected for something when possession of that trait is part of an explanation as to why organisms with it have survived and reproduced more than organisms without that trait. And

\footnotetext{
${ }^{4}$ This objection is normally attributed to (Fodor 1984/1990). For Burge's formulation of it, see (2010, 299).

${ }^{5}$ Not everyone accepts this conclusion. (Fodor 1990) offers a sophisticated version of IT theory, one that strives to make room for the possibility of error.

${ }^{6}$ The most influential TS theories, those of Millikan and Dretske, draw upon etiological theories of biological functions. See Wright (1973); Millikan (1989); Neander (1991); Griffiths (1993); Walsh and Ariew (1996); Buller (1998).
} 
what a trait is selected for-hence, what its function is - is whatever that trait has done to make organisms with that trait "fitter" than organisms without such a trait."

Take a typical example, the human heart. Roughly speaking, hearts have the biological function of pumping blood because they were selected for pumping blood. That means that ancestors of humans with hearts that pumped blood were fitter than ancestors of humans with hearts that did not pump blood; ancestors with hearts that pumped blood reproduced more successfully than ancestors without such hearts. In this sense, pumping blood is what hearts should do. The force of this "should" is differential fitness. In a given environment, all else being equal, ancestors who had hearts that did not pump blood were less adaptive than ancestors who had hearts that do. This implies that if a heart does not pump blood, it is malfunctioning.

TS theories of perceptual representation apply this idea of a function to sensory states. The basic idea is that a perceptual representation is a sensory state that has the function of indicating some state of the environment. ${ }^{8}$ What is it for a sensory state $\mathrm{S}$ of an organism $\mathrm{O}$ to have the function of indicating some state of the environment $E$ ? $S$ has been selected for indicating E. That means that past Os that have had Ss that indicate Es have been fitter than Os that have had Ss that do not indicate Es; Os that have had Ss that indicate Es have survived longer or reproduced more successfully than Os that have lacked such Ss. Thus, indicating E is what S should do. As with the human heart, the force of this "should" is differential adaptiveness. In a given environment, all else being equal, Os that have Ss that do not indicate Es are less fit than Os that have Ss that do indicate Es. This implies that an occurrence of $\mathrm{S}$ is a malfunction if it occurs when no E occurs, or if it is not caused by an E.

For Burge, the key problem with TS theories is that proper and improper functioning is independent of representational success or failure. That is, although functions make room for a sort of error, that sort of error is not the same as representational error or inaccuracy. A sensory state can fulfill its function without being accurate, and it can fail to fulfill its function without being inaccurate. After all, fast but inaccurate sensory systems are often adaptive, just as slow but accurate systems are often maladaptive. ${ }^{9}$ Burge claims that no account of perceptual representation in terms of practicality or usefulness is adequate. ${ }^{10}$ Yet, TS theories are just that sort of theory. They attempt to account for perceptual representation in terms of practical success. For that reason, Burge thinks TS theories fail to account for perceptual representation. ${ }^{11}$

Burge does not hold that TS theories are completely off track. While they fail to state a sufficient condition for perceptual representation, they succeed in stating a necessary condition for it. The necessary condition is that, like all sensory states, genuine perceptual representations have a biologically evolved function of indicating a state of the environment. ${ }^{12}$ But only some such states are genuinely representational. So, at this point, the key question is what else must be

\footnotetext{
${ }^{7}$ This is a rough gloss on functions. For further discussion, see Sober (1993/2000) and Sterelny and Griffiths (1999).

${ }^{8}$ Dretske (1988) is the best example of this specific sort of TS theory. For a contrasting sort of TS theory, see (Millikan 1984).

${ }^{9}$ See, for example, (Trimmer, et al. 2008)

${ }^{10}$ See Burge $(2010,301)$.

${ }^{11}$ As with Burge's criticism of IT theories, not everyone accepts this criticism of TS theories. See (Neander 2004/2012) for a sophisticated defense of TS theories.

12 See Burge $(2010,317)$.
} 
true of a sensory state for it to qualify as a genuine perceptual representation? What distinguishes genuine perceptual representations from mere sensory responses?

\subsection{What does suffice for perceptual representation?}

Burge proposes that perceptual representations differ from mere sensory responses in virtue of having (what he calls) veridicality conditions, conditions under which the state is accurate or true. And he thinks that sensory states manage to have such conditions if they are exercises of (what he calls) constancy mechanisms. In short, if a sensory state is the exercise of a constancy mechanism, then it is a perceptual representation. As Burge puts this point, "Perceptual constancies are the key to understanding the nature of perception" $(2010,349)$; "their presence in a sensory system is necessary and sufficient for the system's being a perceptual system" (2010, 413).

Constancy is a pervasive feature of our perceptual engagement with the world. For instance, if you walk around a square table, viewing it from different angles and distances, the input to your visual system from the light reflected off of the table varies considerably, producing a variety of different retinal images. ${ }^{13}$ On their own, each of these individual images could equally have been produced by a differently shaped table, and the sequence of changing images could equally well have been produced by a table that is itself changing shape. Yet you are nevertheless able to perceive a single table with an unchanging shape; you are able to perceive one, constant shape, not merely a sequence of many, different shapes. This is a case of shape constancy, which is one of several visual constancies, including size, distance, and color constancy. Constancy is also exhibited by non-visual perceptual systems. For instance, as you walk closer to or further from a speaker emitting music at a set volume, you are able to have a constant or non-varying perception of the music's volume. Your perception of the volume of the music does not change across the changing conditions in which you hear it; you are able to perceive it as remaining the same volume, not of it increasing or decreasing in volume. In general, constancy "is nothing more or less than a stability in perceptual response across a range of varying perceptual conditions" (Cohen ***, ***2-3***). ${ }^{14}$

Given that the input to our perceptual system at any given moment underdetermines what caused that input, how do we manage to have a determinate perception of one aspect of this input rather than any of the other aspects? Following Burge, we will call this problem the underdetermination problem. ${ }^{15}$ Constancy requires solving the underdetermination problem.

The underdetermination problem is not easy to solve. As noted, even a simple, regularly shaped object (such as a square table) can produce a tremendous variety of retinal images in

\footnotetext{
${ }^{13}$ This is not to say that we perceive these images on our retinae. Visual experience is produced by the light that is absorbed by our retinae, not the light reflected by our retinae. That said, speaking of the "images on our retinae" is useful shorthand for speaking of the patterns of proximal sensory activity that take place when we visually perceive something. But we should not be misled by this shorthand: we simply do not perceive the curved, inverted images in our retinae.

${ }^{14}$ For Burge's formulation, see Burge (2010, 388). For formulations by other authors, see Palmer (1999, 312 13,723), Byrne and Hilbert (1997, 445), and Goldstein (2009, 218, 410).

${ }^{15}$ For Burge's formulation, see Burge (2010, 344). See also Palmer (1999, 23, 716).
} 
different viewing conditions. Thus, it is tempting to assume that the ability to solve the underdetermination problem must involve a higher-level cognitive capacity for reasoning: a conscious, voluntary, and general ability to think thoughts like SQUARE TABLES SEEN FROM CHAIRS LOOK LIKE TRAPEZOIDS. Although tempting, this assumption is at odds with the dominant view in vision science for the last century and a half. ${ }^{16}$ The dominant view holds that solving the underdetermination problem does not require such higher-level cognitive processes. Instead the underdetermination problem is solved by constancy mechanisms that are unconscious, automatic, and domain-specific. ${ }^{17}$

A constancy mechanism is a capacity to generate a single type of response to varying proximal input in such a way that this response tracks or correlates with some single type of distal stimulus. Constancy mechanisms solve the underdetermination problem by relying on (what Burge calls) "formation principles" that transform proximal input into responses to distal stimuli. ${ }^{18}$ Here is how Burge describes these transformations:

These laws or law-like processes serve to privilege certain among the possible environmental causes over others. The net effect of the privileging is to make the underdetermining proximal stimulation trigger a perceptual state that represents the distal cause to be, in most cases, exactly one of the many possible distal causes that are compatible with (but not determined by) the given proximal stimulation. $(2010,92)$

Formation principles transform proximal input into responses to distal stimuli by privileging some possible causes over others: it receives proximal stimuli as input, which could have been caused by a variety of different distal stimuli, and via the formation principles, produces an output that tracks or correlates with only a much more restricted range of possible distal stimuli. Burge compares formation principles to filters that simply eliminate a host of possible distal stimuli. ${ }^{19}$ Obviously, randomly eliminating possible distal stimuli will not work. Formation principles work - they solve the underdetermination problem - by disregarding unlikely distal stimuli. More exactly, formation principles exploit the fact that because we tend to be in certain specific kinds of environments and tend to move around in them in certain specific kinds of ways, many possible distal stimuli are extremely unlikely to be causes and can simply be discounted. ${ }^{20}$ For example, in our world, there are not very many table-like objects that constantly change shape. Formation principles solve the underdetermination problem by simply disregarding unlikely possibilities.

\footnotetext{
${ }^{16}$ Palmer (1999) is an especially clear introduction to this tradition.

${ }^{17}$ Burge summarizes what he takes the dominant view to involve at Burge (2010, 101-3).

${ }^{18}$ For Burge's formulation, see Burge (2010, 345).

${ }^{19}$ As Burge puts it, "Formation principles describe processes that begin with selective filtering of the initial sensory registration" $(2010,92)$. See also Burge $(2010,274,282,285,371)$. In some respects, filters are a useful metaphor for beginning to explain how formation principles solve the underdetermination problem. However, this metaphor should not be taken too literally or without qualification. Consider, for example, a standard filter, such as a HEPA air filter, which blocks the passage of airborne particles that are larger than 0.3 microns. HEPA filters do this by being presented with airborne particles of a variety of sizes and only allowing those that are smaller than 0.3 microns to pass through. Filters like this are very different from formation principles, because formation principles are not presented with all of the possible distal stimuli. Possibilities, as such, do not exist for formation principles, so it is misleading to say that formation principles literally "filter" some of them out. Nevertheless, such metaphorical talk can be helpful shorthand for the way in which formation principles take an input that is indeterminate between many distal causes and generate an output that tracks just one distal cause.

${ }^{20}$ See Burge $(2010,100)$.
} 
Relying on formation principles, constancy mechanisms solve the under-determination problem without any conscious work or reasoning by perceivers. In Burge's words:

[F]ormation principles describe and explain laws instantiated in transformations in the system. They are not applied in reasoning or cognition, even "implicit" reasoning or cognition, within the system. Thinking of them as applied by the system hyper-intellectualizes the system. $(2010,97)$

Formation principles are not rules that we perceivers entertain and follow, but simply law-like regularities that are exhibited by constancy mechanisms. They do not involve any sort of general ability to reason about how one-and-the-same object will look or sound across varying conditions. Rather, they are specific to individual types of constancies (e.g., shape, color, volume, etc.), automatic, and instantiated independently of any general reasoning ability. In a word, constancy mechanisms are modular. ${ }^{21}$

Burge appeals to constancy mechanisms to explain perceptual representation, and specifically to explain how they have veridicality conditions. Why does Burge think that exercises of constancy mechanisms have veridicality conditions? At bottom, it is because he thinks they distinguish between perceiver-independent facts or properties and perceiverdependent facts or properties; according to him, there is a sense in which they distinguish appearance from reality. ${ }^{22}$ Consider again the visual experience of the shape of the table. For a shape constancy mechanism to solve the under-determination problem in that case, allowing us to have a perception of a square table, rather than a trapezoidal one, the mechanism must be able to distinguish the real, unchanging, perceiver-independent shape of the table from its changing, perceiver-dependent appearances across different viewing conditions.

Burge is not alone in thinking that genuine perceptual representation requires being able to distinguish reality from appearance. For instance, two of Burge's primary opponents, P. F. Strawson and Gareth Evans, also think that perception requires it. As Evans puts this point, genuine perceptual representation involves being able to distinguish between "that of which there is an experience (part of the world) and... the experience of it (an event in the subject's biography)" (Evans 1980/1996, 277). ${ }^{23}$ As Burge sees it, however, making such a distinction does not require general, higher-order, or conscious thinking. Instead, Burge contends that constancy mechanisms do this when they distinguish distal causes from proximal sensory activity. Burge calls this ability “objectification.” In his words:

Objectification lies in marking off states that are as of specific system-independent elements in the environment from states idiosyncratic or local to the perceiver. ... Objectivity is the product of separating what occurs on an individual's sensory surfaces from the significance of those stimulations for specific attributes and particulars in the broader environment. In this way, perception is the product of objectification. $(2010,400)$

\footnotetext{
${ }^{21}$ We ourselves think that the extent to which perceptual constancy mechanisms are modular is more contested in contemporary vision science than Burge himself seems to think, but, for the purposes of this essay, we will follow him in characterizing this as the consensus view. Our own criticisms of Burge do not depend upon contesting the extent to which perceptual constancy mechanisms are modular.

${ }^{22}$ See Burge $(2010,397)$.

${ }^{23}$ Similarly, Strawson argues that genuine perceptual representation involves an ability to draw a "distinction between oneself and one's states on the one hand, and anything on the other hand which is not either oneself or a state of oneself, but of which one has, or might have, experience" (Strawson 1959/2005, 69).
} 
Because an exercise of a constancy mechanism "objectifies"- -because it distinguishes the distal world from proximal effects on the organism - it purports to be of or about some distal stimulus, such as an object's shape. Because it does that, it can be accurate or inaccurate; it can get the distal stimulus right or wrong. Thus, according to Burge, it has veridicality conditions. By contrast, mere sensory systems do not objectify - they cannot distinguish perceiver-independent, distal causes from perceiver-dependent, proximal sensory input. Thus, they do not have veridicality conditions.

\subsection{Burge's Account of Misrepresentation}

If exercises of constancy mechanisms have veridicality conditions, then it must be possible for them to be accurate as well as inaccurate. But what is it for an exercise of a constancy mechanism to be inaccurate?

Here is a short answer. Misrepresentation occurs when a constancy mechanism fails to solve the underdetermination problem because it is exercised in abnormal conditions.

Here is a longer answer. A constancy mechanism has the function of solving the underdetermination problem, of tracking some single distal cause across changes in sensory inputs. It relies upon formation principles to do this. Formation principles determine these distal causes by privileging certain possible causes over a host of possible causes. In particular, they privilege the statistically likely, or normal, distal causes of sensory inputs. However, since the actual cause of a type of sensory input is not always the normal cause of that type of input, formation principles are fallible. ${ }^{24}$ Thus, a constancy mechanism can respond as though the cause of the input is something normal when in fact the cause is not something normal. In other words, Burge holds that a constancy mechanism can identify something as the distal cause of a sensory input that is not the cause of that input. Since the function of the constancy mechanism is to determine the distal cause of an input to the sensory system, in these cases, the constancy mechanism has erred; it has gone wrong. For that reason, Burge contends that it is possible for constancy mechanisms to misrepresent. ${ }^{25}$

In sum, Burge holds that perceptual misrepresentation occurs under the following three conditions: there is a constancy mechanism that has the function of responding to a type of distal cause across changes in sensory input; that constancy mechanism is exercised in a particular instance; in this particular instance, the conditions under which it is exercised are abnormal, leading it to be exercised in response to something other than the type of distal cause that it has the function of responding to, thereby leading it to fail to solve the underdetermination problem.

\section{$\underline{\text { 2.4 Summary }}$}

To see what is distinctive about Burge's view, compare it with the views that he rejects. Burge holds that Information-Theoretic theories do not make room for the possibility of error; as such,

\footnotetext{
${ }^{24}$ For Burge's formulation, see $(2010,346)$.

${ }^{25}$ See Burge $(2010,98,344)$
} 
they do not make room for the possibility of misrepresentation. He admits that Teleosemantic theories make room for the possibility of error, but he alleges that this sort of error is not the same as misrepresentation. Failure to fulfill a function is failure to do what's useful; and that need not have anything to do with accuracy or inaccuracy. Burge nevertheless holds that Teleosemantic theories provide a necessary condition for perceptual representations: like all sensory states, genuine perceptual representations have the function of indicating a state of the environment $(2010,317)$. It is only when such sensory states have the more specific task of solving the underdetermination problem that they count as representations, states with veridicality conditions. ${ }^{26}$ In the next section, we argue against this aspect of Burge's account.

\section{Constancy is Not Enough for Perceptual Representation}

In this section, we argue there are two problems for Burge's account. First, if mere sensory responses are not representational, then neither are exercises of constancy mechanisms, since the differences between them do not suffice to imply that one is representational and the other is not. Second, taken by themselves, exercises of constancy mechanisms are only derivatively representational, so merely understanding how they work is not sufficient for understanding what is required for something, in itself, to be representational.

\subsection{Proximity is Irrelevant}

The gist of our first criticism is that mere sensory experiences and exercises of constancy mechanisms stand or fall together, and since Burge gives good reasons for holding that mere sensory experiences are not representational, he should not hold that exercises of constancy mechanisms are either.

For Burge, exercises of constancy mechanisms and mere sensory responses are similar in that they both have indicator functions (that is, the function of indicating something). Having a function brings with it the possibility of malfunction. Accordingly, a first key similarity between exercises of constancy mechanisms and mere sensory responses is that they both allow for the possibility of misindication. Consider, for example, a paradigmatic example of a mere sensory response: the shiver response. ${ }^{27}$ Warm-blooded mammals shiver in response to hypothermia. Shivering has the function of indicating that the organism's core body temperature has dropped below the level required for normal metabolic activity. It is possible, therefore, for shivering to misindicate a drop in core body temperature (if it is triggered in the absence of such a drop). Given this, it would be a mistake to think that exercises of constancy mechanisms differ from mere sensory responses on the grounds that exercises of constancy mechanisms are uniquely capable of misindication. This is something they both share.

There is a second, subtler similarity between exercises of constancy mechanisms and mere sensory responses. The possibility of misindication requires a difference between (a) being in some state or exhibiting some response, and (b) correctly or incorrectly being in that state or

\footnotetext{
${ }^{26}$ See Burge $(2010,303,309)$.

${ }^{27}$ See, for instance, (Jensen 2001).
} 
exhibiting that response. For example, in the case of the shiver response, whether the organism is shivering must be independent of whether the shivering is correctly or incorrectly indicating a drop in core body temperature. The fact that a warm-blooded mammal is shivering does not by itself imply that its core body temperature has dropped. So, it would be a mistake to think that this distinction between (a) and (b) holds uniquely for exercises of constancy mechanisms. The distinction holds equally well for mere sensory responses.

Let us return now to why Burge supposes that mere sensory responses differ from exercises of constancy mechanisms. Burge thinks that exercises of constancy mechanisms are distinctive because they distinguish between appearance and reality; they "objectify." By this, he means that exercises of constancy mechanisms distinguish proximal effects on the organism from the distal causes of those effects. For instance, an exercise of a shape-constancy mechanism indicates (and has the function to indicate) the shape of a distal object, not the shape of the retinal image caused by the object. Mere sensory responses do not do that; they only have the function to indicate proximate stimuli. For instance, the shiver response has the function to indicate a proximal state of the organism, not a distal state in the organism's environment. So, for Burge, the key difference between exercises of constancy mechanisms and mere sensory responses is that exercises of constancy mechanisms have the function of indicating distal stimuli, whereas mere sensory states have the function of indicating proximal stimuli.

Burge then contends that this difference is decisive for the issue of perceptual representation. Because exercises of constancy mechanisms objectify and thereby indicate distal stimuli, not proximal stimuli, they have accuracy conditions and are thereby representational. We think Burge's reasoning here is mistaken. Our key question for Burge is: why think a difference in proximity is what matters for having accuracy conditions?

Burge's argument appears to be this.

1. If something has the function to indicate distal stimuli, as opposed to proximal stimuli, then it is able to distinguish between perceiver-independent and perceiver-dependent states of affairs.

2. Being able to distinguish between perceiver-independent and perceiver-dependent states of affairs is akin to distinguishing between reality and appearance.

3. Being able to distinguish reality from appearance suffices for having accuracy conditions.

4. Exercises of constancy mechanisms have the function to indicate a distal stimulus.

5. So, they have accuracy conditions.

While this argument is valid, its second premise is dubious. What reason is there for thinking that something that has the function of indicating a distal stimulus distinguishes between appearance and reality? Burge might contend that something that has the function of indicating a distal stimulus (e.g., the shape of a distal object) rather than a proximal stimulus (e.g., the shape of a retinal image) is able to distinguish distal things from proximal things. That might be true, but it does not follow that such a thing distinguishes appearance from reality (or that it has the function to do so). Distinguishing between proximal and distal things is not the same as - and does not suffice for-distinguishing appearance from reality. It merely involves being able to distinguish two, equally real, things: proximal stimuli and distal stimuli. Furthermore, the fact that the organism or system's response to the distal stimuli is mediated by the proximal stimuli, does not make either sorts of stimuli any less real. In short, it is a mistake to 
think that the shape of a retinal image is merely apparent; the shape of the retinal image is just as real as the shape of the object that causes it.

Underlying the idea that exercises of constancy mechanisms distinguish between appearance and reality is a further mistake. It requires the proximal stimulus (e.g., the retinal image) to be an appearance. But for something to be an appearance, it must be a representation; it must appear to represent things as being a certain way. ${ }^{28}$ However, Burge rightly does not think that proximal stimuli (such as retinal images) are themselves representational; he does not think that they have accuracy conditions. But then he cannot allow that proximal stimuli are appearances. So, the idea that exercises of constancy mechanisms distinguish between appearance and reality is deeply problematic.

Why else might one think that the proximity of a stimulus matters? One might think it matters because exercises of constancy mechanisms are mediated responses, whereas mere sensory responses are immediate responses. That is, one might contend that an exercise of a constancy mechanism is representational because there is an intermediate state between an exercise of a constancy mechanism and the state of affairs that it has the function of indicating. For instance, consider once again an exercise of a shape-constancy mechanism. One might contend that what matters is that there is a retinal image that mediates the connection between an exercise of a constancy mechanism and the shape that the exercise has the function of indicating. But why think the presence of an intermediary matters? As we have just noted, the intermediary is not, in any sense, representational. Even Burge himself does not think that it is.

One might think instead that the crucial difference is that exercises of constancy mechanisms have the function to indicate something despite variation in an intermediary. For instance, an exercise of a shape-constancy mechanism has the function to indicate the shape of a distal object despite variation in the retinal images caused by the object. Again, why think such variation matters? Would exercises of a shape-constancy mechanism cease to be representational if the proximal retinal did not vary? That seems implausible. Although not decisive, it helps to think about cases such as spheres, whose retinal images do not vary across changes in line of sight. Would that suffice to show that perceptions of spheres are not representational? Are perceptions of cubes representational, while perceptions of spheres not? In short, it seems implausible to think that whether or not the intermediate state varies is what makes the difference between representational sensory responses and non-representational sensory responses.

Summing up, Burge says there is a difference in kind between two sorts of sensory responses; one sort is representational, the other is not. One sort is representational because it has the function of indicating a distal stimulus; the other is not representational because it has only the function of indicating a proximal stimulus. We have claimed that this difference does not suffice for the first sort of sensory response to be representational. The proximity of a stimulus seems to be irrelevant to whether or not a response to that stimulus is representational. We acknowledge that there are further differences between exercises of constancy mechanisms and mere sensory responses. But these differences do not seem to suffice for exercises of constancy

\footnotetext{
${ }^{28}$ This claim is neutral with regard to debates over whether appearances are exhausted by their representational
} contents. 
mechanisms to be representational. Thus, no good reason has been given for thinking that merely being an exercise of a constancy mechanism suffices for having accuracy conditions. (Perhaps such exercises do have accuracy conditions when they are suitably involved in larger systems.)

\subsection{Constancy Mechanisms Do Not Have Original Intentionality}

The gist of our second criticism is that exercises of perceptual constancy mechanisms are only derivatively representational, whereas what is needed for Burge's proposal to succeed is for them to be non-derivatively representational. To spell out this criticism, we introduce a pair of distinctions. The first is John Haugeland's important and influential distinction between original and derivative intentionality. The second is our own distinction between original and derivative beholdenness to norms of accuracy. In brief, we argue that perceptual constancy mechanisms are only derivatively representational because they are only derivatively beholden to norms of accuracy.

The first distinction is Haugeland's distinction between original and derivative intentionality. "Intentionality" is just another word for being about something, in precisely the way that representations are about something. Here is how Haugeland introduces this distinction:

Intentionality... is not all created equal. At least some outward symbols (for instance, a secret signal that you and I explicitly agree on) have their intentionality only derivatively - that is, by inheriting it from something else that has that same content already (such as the stipulation in our agreement). And, indeed, the latter might also have its content only derivatively, from something else again; but, obviously, that cannot go on forever.

Derivative intentionality, like an image in a photocopy, must derive eventually from something that is not similarly derivative; that is, at least some intentionality must be original (nonderivative). And clearly then, this original intentionality is the primary metaphysical problem; for the possibility of delegating content, once there is some to delegate, is surely less puzzling than how there can be any in the first place. $(1998,129)$

In short, Haugeland's distinction is between intentionality that is derived from something else, and intentionality that is not so derived —or (as he calls it) "original."

We take for granted that in aiming to solve the problem of perceptual representation, Burge aims to show how acts of perception have original intentionality, not merely derivative intentionality. The problem of perceptual representation is a problem precisely because it requires making sense of the original intentionality of acts of perception. Our second criticism of Burge is based upon the claim that exercises of constancy mechanisms do not have original intentionality, but at most derivative intentionality. Our argument for this claim has two steps: first, original intentionality requires being beholden to norms of accuracy in a certain way; second, constancy mechanisms are not beholden to norms of accuracy in that way.

To understand the first step of our argument, think about the phenomena of being beholden to norms in general. Consider two cases. In the first case, Nat says, "The window should be shut." In this case, the window is being held to a norm. It should be shut. But the window, in itself, is not capable of modifying its own behavior in response to a failure to abide by this norm. Of course, something else can modify the window's behavior (say, if Wyeth shuts 
it), but the window alone cannot do it. As such, the window is only derivatively beholden to this norm. In the second case, Nat quietly says to himself, "I should shut my mouth." Here Nat is being held to a norm. And it is he that is holding himself to it, in thinking and saying it. (Whether he complies is another matter.) In this case, he is non-derivatively or originally beholden to a norm.

In general terms, a system $\mathrm{S}$ is non-derivatively or originally beholden to a norm $\mathrm{N}$ if and only if $\mathrm{S}$ can modify its own behavior in response to failures to abide by N. S is derivatively beholden to a norm $\mathrm{N}$ if and only if something other than but not $\mathrm{S}$ itself is required to modify $\mathrm{S}$ 's behavior in response to S's failures to abide by $\mathrm{N}$.

For a system to be non-derivatively or originally beholden to a norm of accuracy, therefore, it must be possible for that system to modify its behavior when it is inaccurate. Accordingly, we think that original intentionality - hence the sort of intentionality that Burge thinks perceptual representations have-requires original beholdenness to norms of accuracy.

To understand the second step of our argument, consider what constancy mechanisms can and cannot do. Burge is right to be impressed by constancy mechanisms. He is also right to think that earlier discussions of perceptual representation - by both philosophers and psychologistsfailed to see what is impressive about constancy mechanisms, because they did not fully appreciate how constancy mechanisms involve sensory states that have the function of responding to distal stimuli across changes in sensory input. Peter Schulte provides a useful summary of how our understanding of constancy mechanisms has shifted in this respect:

In the early days of neurological research on frogs, scientists thought that certain retinal ganglion cells, which respond strongly to the movement of small, dark spots on the retina, were almost solely responsible for generating the frog's feeding response (Lettvin et al. 1959; Maturana et al. 1960). These neurons were colloquially called "bug detectors."

But it soon became clear that matters were not that simple. Further research showed that frogs do not respond to all small, dark spots moving on their retina. If these spots are caused by large objects that are far apart, frogs usually show no feeding response. And conversely, they do respond to large, dark spots if these spots are caused by small objects that are very close. In other words, frogs respond to the real size of objects, not to the size of retinal stimuli (Ingle 1968; Ingle and Cook 1977). ${ }^{29}(2012,489)$

We think that this summary is doubly useful. First, it is useful because it perfectly captures what Burge thinks is significant about constancy mechanisms for understanding perceptual representation. Second, it is useful because, like Burge, it glaringly overlooks a crucial feature of constancy mechanisms. That feature is under-constancy. ${ }^{30}$

Under-constancy is simply the fact that well-functioning constancy mechanisms do not always track the same distal stimuli across changes in proximal stimuli. ${ }^{31}$ Size constancy

\footnotetext{
${ }^{29}$ For a useful summary of research on frog size-distance constancy mechanisms, see Ingle (1998).

${ }^{30}$ For a useful historical survey of studies of under-constancy, see Wagener (2012).

${ }^{31}$ Palmer says that under-constancy "is incomplete perceptual constancy in which the perception is a compromise between proximal and distal matches" (1999, 733, [emphasis in original]). Palmer also characterizes underconstancy as the way in which constancy mechanisms exhibit "systematic deviations from accurate perceptions of objective properties in the direction of proximal image matches" (1999, 314). As will become clear, we think that
} 
mechanisms, for instance, often systematically under- and over-estimate the sizes of objects, in predictable ways. That is, built into the way in which constancy mechanisms track distal stimuli, is the fact that they often end up tracking a range of distal stimuli, due to the effect of differences in proximal stimuli on their responses. This means that it is false to say, as Schulte does, that size-distance constancy mechanisms "respond to the real size of objects, not to the size of retinal stimuli" (our emphasis). It is false because size-distance constancy mechanisms only respond to the real size of objects some of the time. At other times, their responses are affected by the size of retinal stimuli. As Mazviita Chirimuuta puts this point in general terms, "constancy is not perfect" $(2015,55)$. In sum, Burge over-emphasizes the degree to which constancy mechanisms exhibit constancy, thereby overlooking the effect that differences in proximal stimuli sometimes have on the exercise of constancy mechanisms. ${ }^{32}$

Under-constancy exposes a problem for Burge's account because it reveals how constancy mechanisms are not beholden to norms of accuracy in the right sort of way to produce genuine perceptual representations. What is the right sort of way? Burge himself does not raise this question, because he does not distinguish two important ways in which representations can be beholden to norms of accuracy. This is a fatal oversight for Burge's theory, because any talk of representations (perceptual or otherwise) being beholden to norms of accuracy is ambiguous.

Since constancy mechanisms are unable to respond to failures of constancy, they are unable to correct themselves in response to errors. They simply do not have any ability to modify their responses in response to under-constancy. For a sensory state to be a genuine perceptual representation, however, we think it would have to be part of a system that is non-derivatively beholden to a norm of accuracy. Constancy mechanisms by themselves are not such a system. ${ }^{33}$

To prevent misunderstanding, two qualifications are in order.

First, under-constancy in itself is not the problem. It is how constancy mechanisms respond (or do not respond) to under-constancy that is the problem. We are not claiming that representational systems must be perfect, or that they must always be accurate. Far from it: we

Palmer's use of "accurate" in this characterization is ambiguous between two readings of what it is for a sensory state to be beholden to a norm of accuracy: namely, between being originally and derivatively beholden.

${ }^{32}$ Another way to put this point is to note that many perceptual illusions are the direct result of the ways in which constancy mechanisms solve the underdetermination problem. Here's how Palmer puts this point:

Somewhat surprisingly, the same processes that usually result in constancy - that is, veridical perception-sometimes produce illusions. The reason is that [...] veridical perception of the environment often requires heuristic processes based on assumptions that are usually, but not always, true. When they are true, all is well, and we see more or less what is actually there. When these assumptions are false, however, we perceive a situation that differs systematically from reality: that is, an illusion. In several cases we will find that in the perception of object properties, constancy and illusion are therefore opposite sides of the same perceptual coin. (1999, 313)

Once again, as with "accurate", we think Palmer's use of words like "veridical" and "true" is ambiguous (see the previous footnote). It is understandable that Palmer would use such words, because they are far-and-away the easiest way to describe constancy and under-constancy. Nonetheless, we think using words like "accuracy", "veridical", and "true" - without disambiguating what one might mean by them — can be misleading.

${ }^{33}$ This is not to say that they cannot be part of such a system. Burge's account of genuine perceptual representation treats constancy mechanisms as modular, self-contained, representational systems. Our goal in this essay is to show that such an account fails. We ourselves think that constancy mechanisms are often parts of representational systems. We describe the conditions under which this is the case in Adams and Maher (2012). 
agree with Burge that genuine representational systems must make room for the possibility of misrepresentation. Rather, we are saying that such systems must be able to respond to errors in order for such errors to count as inaccuracies.

Second, it is not that constancy mechanisms do not, in fact, respond to under-constancy that is the problem. It is their inability to respond to under-constancy that is the problem. We are not saying that representational systems must always respond to inaccuracies. We agree with Burge that representational error is in principle independent of practical consequences (see $\S 2.1 .2$. above). So, we agree with Burge that a system can misrepresent how things are without that misrepresentation leading to any actual consequences whatsoever. Furthermore, it would be absurd to think that for a representational system to be beholden to a norm of accuracy, it must always correct its misrepresentations, for two reasons. First, the fact that a system is in error need not entail that it recognizes that it is in error. Second, it would imply that uncorrected misrepresentations are not representations. Speaking generally, the fact that a system is beholden to a norm does not imply that it must actually correct failures to abide by that norm. Again, our claim is only that a system must be able to respond to errors in order for such errors to count as inaccuracies.

Summing up, our second criticism of Burge is that exercises of constancy mechanisms do not suffice for perceptual representation for they do not have original intentionality, for they are only derivatively beholden to norms of accuracy. Constancy mechanisms are not able to correct themselves in response to their own failures to be accurate.

\section{Conclusion}

In this essay, we have summarized Tyler Burge's recent proposal for solving the problem of perceptual representation and offered a pair of criticisms of it. We close by spelling out why we think that the failure of Burge's account is, nevertheless, a very good starting point for solving the problem of perceptual representation.

Exercises of constancy mechanisms, in themselves, do not have veridicality conditions. Something more is needed. But what?

Much of Burge's rhetoric in Origins of Objectivity makes it seem as though the only alternative to his view of perceptual representation is a hyper-intellectualized view, according to which genuine perceptual representation requires that a perceiver be able to represent, to herself, the conditions under which her perceptions would be accurate or inaccurate. ${ }^{34}$ This is, selfevidently, an individualistic and intellectualized view of perceptual representation, one that would, most likely, imply that only humans are capable of genuine perceptual representation. But, following John Haugeland, we think that it is a mistake to assume that the only way in which exercises of constancy mechanisms might be supplemented (in order to produce genuine

\footnotetext{
${ }^{34}$ Burge refers to this sort of hyper-intellectualized account of perceptual representation as Individual Representationalism, the view that "an individual cannot empirically and objectively represent an ordinary macrophysical subject matter unless the individual has resources that can represent some constitutive conditions for such representation" $(2010,13)$.
} 
perceptual representations) is for a perceiver to be able to represent, to herself, the veridicality conditions of her perceptions. There are alternatives that do not have this manifestly individualist and intellectualist form. To see one, consider the case of vervet monkeys. Vervet monkeys are social animals, living in troops, who use a system of alarm calls to alert the troop to different types of threats, thereby eliciting in the troop different types of evasion responses. ${ }^{35}$ It is widely accepted that vervets are genetically predisposed to make such calls as well as respond to them. But there seems to be some evidence that adult vervets train their infants to make such calls only in response to actual predators, by punishing incorrect calls and rewarding correct calls. ${ }^{36}$ If this evidence is correct, then it suggests that vervet monkeys might have visual experiences that are non-derivatively beholden to norms of accuracy. As such, these experiences might be genuine perceptual representations. Whether this is, in fact, true of vervet monkeys is an open question that deserves to be examined at length (in another essay). But the fact that it might be true shows that Burge's rhetoric relies on a false dichotomy. There are not only two options: Burge's view or a hyper-intellectualized view.

The failure of Burge's account is not a dead end. Our critique is compatible with thinking that exercises of constancy mechanisms, when part of larger systems, suffice for perceptual representation. In particular, it is compatible with thinking that exercises of constancy mechanisms, when part of a system of social interactions that are non-derivatively beholden to norms of accuracy, suffice for the resulting sensory states to be genuine perceptual representations. Thus, we think our critique points the way forward in thinking about the problem of perceptual representation. The key question now is this: what more is needed, beyond exercises of constancy mechanisms, for sensory states to be non-derivatively beholden to norms of accuracy?

\section{Bibliography}

Adams, Zed and Chauncey Maher. "Cognitive Spread: Under What Conditions Does the Mind Extend Beyond the Body?" European Journal of Philosophy 20 (4):420-438, 2012.

Bradbury, J, and S. Vehrencamp. Principles of Animal Communication. 2nd. Sunderland, MA: Sinauer Assoc., 2011.

Brandom, Robert. Making it Explicit. Cambridge, MA: Harvard University Press, 1994.

Buller, D. "Etiological theories of function: a geographical survey." Biology and Philosophy 13, no. 4 (1998): 505-527.

Burge, Tyler. Origins of Objectivity. New York: Oxford University Press, 2010.

Byrne, Alex, and David Hilbert. Readings on Color. Vol. 2. Cambridge, MA: MIT Press, 1997.

Caro, T. M., and M. D. Hauser. "Is there teaching in nonhuman animals." Quarterly Review of Biology 67 (1992): 151-174.

Chirimuuta, Mazviita. Outside Color: Perceptual Science and the Puzzle of Color in Philosophy. Cambridge, MA: MIT Press, 2015.

Chisholm, Roderick. Perceiving. Ithaca: Cornell University Press, 1957.

\footnotetext{
${ }^{35}$ See Seyfarth, Cheney, and Marler (1980) and Bradbury and Vehrencamp (2011).

${ }^{36}$ See Caro and Hauser (1992) for a summary of the possible evidence; but see Thornton and Raihani (2008) for some reservations.
} 
Cohen, Jonathan. "Perceptual Constancy." In Oxford Handbook of Philosophy of Perception, edited by Mohan Matthen. OUP, *****.

Cummins, Robert. Meaning and Mental Representation. Cambridge, MA: MIT Press, 1989.

Dennett, Daniel. “Intentional Systems." Journal of Philosophy 68 (1971): 87-106.

Dretske, Fred. Explaining Behavior. Cambridge, MA: MIT Press, 1988.

-. Knowledge and the Flow of Information. Cambridge, MA: MIT Press, 1981.

Evans, Gareth. "Things Without the Mind." In Collected Papers, by Gareth Evans. New York: Clarendon, 1980/1996.

Fodor, Jerry. A Theory of Content and Other Essays. Cambridge, MA: MIT Press, 1990.

Fodor, Jerry. "Semantics, Wisconsin Style." In A Theory of Content, by Jerry Fodor. Cambridge, MA: MIT Press, 1984/1990.

Godfrey-Smith, Peter. "Indication and Adaptation.” Synthese 92, no. 2 (1992): 283-312.

Goldstein, E. Bruce. Sensation and Perception. 8th. New York: Wadsworth, 2009.

Grice, H.P. "Meaning.” The Philosophical Review 66 (1957): 377-88.

Griffiths, Paul. "Functional analysis and proper functions." British Journal for the Philosophy of Science 44, no. 3 (1993): 409-422.

Haugeland, John. "The Intentionality All-Stars." In Having Thought, by John Haugeland, 127170. Cambridge, MA: Harvard University Press, 1990/1998.

—. "Truth and Rule-following." In Having Thought, by John Haugeland, 305-361. Cambridge, MA: Harvard University Press, 1998.

Hemingway, A. "Shivering." Physiological Reviews 43, no. 3 (1963): 397-422.

Ingle, David. "Perceptual constancies in lower vertebrates." In Perceptual Constancy: Why Things Look as They Do, edited by V. Walsh and J. Kulinkowski. New York: Cambridge University Press, 1998.

James, William. Pragmatism. New York: Dover, 1907/1995.

Jensen, Claus. Temperature Regulation in Humans and Other Mammals. Berlin: Springer, 2001.

Millikan, Ruth. "Biosemantics." The Journal of Philosophy 86, no. 6 (1989): 281-297.

—. "In Defense of Proper Functions." Philosophy of Science 56, no. 2 (1989): 288-302.

—. Language, Thought and Other Biological Categories. Cambridge, MA: MIT Press, 1984.

Neander, Karen. "Misrepresenting and Malfunctioning." Philosophical Studies 79, no. 2 (1995): 109-141.

- . "Teleological Theories of Mental Content." Stanford Encyclopedia of Philosophy. 2004/2012. http://plato.stanford.edu/entries/content-teleological/ (accessed September 1, 2012).

Neander, Karen. “The Teleological Notion of 'Function'.” Australasian Journal of Philosophy 69 (1991): 454-468.

Palmer, Stephen. Vision Science. Cambridge, MA: MIT Press, 1999.

Schulte, Peter. "How Frogs See the World." Philosophia 40, no. 3 (2012): 483-496.

Seyfarth, R.M., D.L. Cheney, and P. Marler. "Monkey responses to three different alarm calls: Evidence for predator classification and semantic communication." Science 210 (1980): 801-803.

Shannon, Claude, and Warren Weaver. The Mathematical Theory of Communication. UrbanaChampaign, IL: University of Illinois Press, 1949.

Sober, Elliot. Philosophy of Biology. 2nd. Boulder, CO: Westview Press, 1993/2000. 
Sterelny, Kim. The Representational Theory of Mind. New York: Blackwell, 1991.

Sterelny, Kim, and Paul Griffiths. Sex and Death. Chicago: University of Chicago Press, 1999.

Strawson, P.F. Individuals. New York: Routledge, 1959/2005.

Thornton, A., and N.J. Raihani. "The evolution of teaching." Animal Behavior 75 (2008): 18231836.

Trimmer, P., et al. "Mammalian choices: combining fast-but-inaccurate and slow-but-accurate decision-making systems." Trimmer P., Houston A., Marshall J., Bogacz R., Paul E., Mendl M., McNamara J.. (Proc Biol Sci 275, no. 1649 (2008): 2353-61.

Wagner, M. "Sensory and Cognitive Explanations for a Century of Size Constancy Research." In Visual Experience: Sensation, Cognition, and Constancy, edited by G. Hatfield and S. Allred. New York: Oxford University Press, 2012.

Walsh, D.M., and A. Ariew. "A Taxonomy of Functions." Canadian Journal of Philosophy 26, no. 4 (1996): 493-51 